\title{
A review on conventional and novel topical ocular drug delivery system.
}

\author{
Mohamad Faeznudin Rozi ${ }^{1}$, Awis Sukarni Mohmad Sabere ${ }^{1^{*}}$
}

\section{ABSTRACT}

Ocular drug delivery is a very challenging area for ophthalmologists and drug delivery scientists due to the structural and barrier complexity of the eye. Barriers such as different layers of cornea, sclera, conjunctival blood flow, and tear dilution limit the efficacy of drug delivery to the anterior part of the eye in addition to more barriers present to the posterior part. Due to these, scientists have designed and studied various delivery systems to increase drug delivery and treatment efficacy to the eye. Among conventional ocular drug delivery systems, ophthalmic solution or eye drop is widely used and preferred by consumers. Conventional dosage forms available in the market are emulsion, suspension, ointment and polymeric gels. Several ocular formulations such as nanoformulations, liposomes, ocular inserts, and ocular mini-tablets are also being widely studied as future treatments to improve ocular drug delivery and as an alternative to conventional drug delivery. This review intends to summarise several conventional and novel topical formulations for ocular drug delivery.

\section{ARTICLE HISTORY:}

Received: 4 August 2020

Accepted: 28 October 2020

Published: 8 January 2021

\section{KEYWORDS:}

Drug delivery system, ocular drug delivery, ophthalmic solutions.

\section{HOW TO CITE THIS}

ARTICLE:

Rozi, M. F. \& Sabere, A.S. M. (2021). A review on conventional and novel topical ocular drug delivery system.

Journal of Pharmacy, 1(1), 19-26.

\section{*Corresponding author:}

Email address: awissabere@iium.edu.my Tel:+609 5714931, Fax: +09 5716775

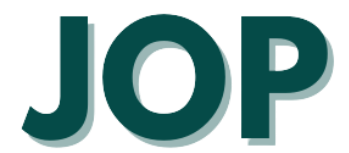

\section{Authors' Affiliation:}

Department of Pharmaceutical Chemistry, Kulliyyah of Pharmacy, International Islamic University Malaysia (IIUM), Jalan Sultan Ahmad Shah, 25200 Kuantan, Pahang, Malaysia 


\section{Introduction}

Eyes are one of the important organs in a human body. As an organ of sense, it allows humans to observe and interact with their surroundings. Generally, the eyeball is divided into two parts, namely the anterior and the posterior segments. The anterior segment of the eyeball contains the cornea, iris, lens, conjunctiva, ciliary body and aqueous humour while the posterior segment consists of sclera, choroid, retinal pigment epithelium, neural retina, optic nerves and vitreous humour (Monkhouse, 2007), as illustrated in Figure 1. Even though the eyeball has many structures, only the anterior part is exposed. The remaining structure is covered and protected by the orbit in which the eyeball is situated. The small exposed portion of the eye is prone to various infections even though it has several protective mechanisms such as the eyelashes, the eyelids and tears (Tortora \& Derrickson, 2015). However, eye diseases are not only limited to infections. Other diseases such as glaucoma, cataract, and allergic conjunctivitis can also inflict the eyes. Thus, a treatment is needed whenever infections or diseases are present in the eyes.

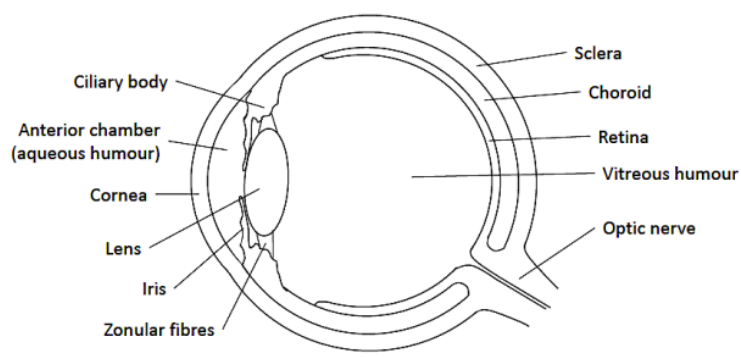

Figure 1 Human eye anatomy.

For the treatment, topical instillation of the agents is the preferable approach as it is easy, convenient and noninvasive. Eye drops are the most commonly used conventional topical ophthalmic dosage form due to the ease of administration and patient compliance (Patel, Cholkar, Agrahari, \& Mitra, 2013). However, it is less effective in certain situations or treatments due to the very low ocular bioavailability and low drug permeation into the ocular tissue as a result of tear turnover, nasolacrimal drainage, and blinking. These barriers have been taken into consideration in increasing the effectiveness of the topical ophthalmic dosage form. (Souza, Dias, Pereira, Bernardi, \& Lopez, 2014). As a result, various conventional and novel drug delivery systems have been developed and studied such as emulsion, suspension, ointment, and one that uses the technological development of lipid-based system and polymeric system.

\section{Conventional topical ocular drug delivery system}

Nowadays, there are several types of ocular drug delivery system in the market including ophthalmic eye drops which are highly used by patients. Others are emulsion, suspension, ointment and polymeric gel preparation.

\section{Eye Drops}

Topical eye drops are the most convenient, noninvasive and patient compliant among topical eye preparations. However, according to Pahuja, Arora, \& Pawar (2012), there are a few barriers encountered by eye drops in treatments. Based on the study, a large number of patients faced difficulties in instilling the drops. Besides, the tear drainage that increases with the volume of eye drops can lead to the loss and dilution of the solution. Other than that, the amount of the drug absorbed into the ocular tissue cannot be estimated due to the limited holding capacity of the eye pocket.

Benzalkonium chloride, which is commonly used as preservative, may also cause several problems such as the peeling of the corneal epithelium cells at their borders which inhibits the growth of the cells and enlarges the intercellular spaces in the superficial cells of the cornea (Pahuja et al., 2012). Even though Ghate \& Edelhauser (2006) stated that benzalkonium chloride could improve the corneal permeability of various drugs, the negative side effects caused by it should not be ignored. Due to these limitations, Patel et al. (2013) suggested the use of viscosity enhancer to increase the contact time, permeation enhancer to increase the uptake of active ingredient, and cyclodextrin as a carrier for hydrophobic molecules to increase the bioavailability of the topical eye drop.

\section{Emulsion}

The interest in using emulsion in the past has been resuscitated by submicron emulsion (ranged between 0.1 $\mu \mathrm{m}$ and $0.3 \mu \mathrm{m}$ ) with non-ionic surfactant to increase its stability (Ghate \& Edelhauser, 2006). Patel et al. (2013) stated that emulsion-based formulation could enhance both the solubility and the bioavailability of ocular drugs. Generally, there are two types of emulsion that are already available in the market as a vehicle for active pharmaceutical ingredients, namely oil in water $(\mathrm{o} / \mathrm{w})$ and water in oil (w/o) emulsions (Vandamme, 2002). Among these two emulsions, $(\mathrm{o} / \mathrm{w})$ type is preferable as it is less irritating to the eye and has better ocular tolerance. According to Liang et al. (2008), emulsion-based formulation can offer several benefits in ocular formulation such that it improves the precorneal residence time, enhances the drug's corneal permeation, increases the bioavailability, and it has sustained-release properties. Precorneal residence time can also be improved by using emulsion with chitosan as its surface coating. This is based on the pharmacokinetic study done by Yamaguchi et al. (2009) using chitosan-coated emulsion in comparison to non-coated emulsion on the eyes of male albino rabbits. The results showed improvement on the emulsion mean residence time (1.5 times) and half-life (1.8 times) of the 
drug in comparison to non-coated emulsion.

However, ophthalmic emulsions come with their own limitations. They have low stability and are prone to various types of instability phenomena such as flocculation, coalescence and creaming (Aldrich et al., 2013). Flocculation occurs when the dispersed phase comes out from the suspension and forms flakes. Coalescence is another instability process by which the dispersed droplets in the suspension are continuously combined to form larger droplets. Other than that, one phase in the emulsion can migrate either to the top or the bottom depending on their relative densities, forming a separated layer between the two phases known as creaming. Thus, the study suggested the use of surfactants to improve the kinetic stability of the emulsion products.

\section{Suspension}

Suspension can be defined as a dispersion of finely insoluble active pharmaceutical ingredients in a solvent (Patel et al., 2013). In other words, it is a concentrated solution of active pharmaceutical ingredients. This type of ocular drug delivery system has several benefits over ophthalmic drops. The main benefit is that it can improve the drug's contact time and duration of action due to the insoluble suspension that retains in the precorneal pocket instead of being washed away or diluted by the tear. The improvement of the duration of the drug action is also due to the different particle sizes of the suspended particles. The small particles will replenish the absorbed drug while the large particles will be retained in the precorneal pocket and undergo slow dissolution (Remington, 2011). According to Ghate \& Edelhauser (2006), prednisolone acetate suspension is the most effective to go across the cornea and suppress corneal inflammation compared to prednisolone phosphate solution. There were also four weeks randomised, double-blinded, multicentre phase II clinical trial done on $1 \%$ and $2 \%$ repabimide suspension over placebo. This trial revealed that both suspensions are well tolerated and effective in treating dry eye compared to the placebo (Kinoshita et al., 2012). In addition, higher concentration of suspension was found to be more effective than one with a lower concentration.

Despite all the benefits, suspension also has several drawbacks. For example, due to the high viscosity of TobraDex $^{\circledR}$, Scoper et al. (2008) experimented by reducing the viscosity and improving its pharmacokinetics along with bactericidal activity. This resulted in a new suspension formulation, TobraDex $\mathrm{ST}^{\circledR}$, which showed better formulation characteristics, pharmacokinetics, bactericidal characteristic and patient compliance. Another drawback of suspension formulation is that it needs to be shaken to reach the required dosage level. This will decrease patient compliance and vary the dosage of the drug delivered to the eye. Ghate \& Edelhauser (2006) stated patient compliance as a limiting factor in ocular drug efficacy as the efficacy will increase with dosing frequency. With low patient compliance, the efficacy of the suspension might also be affected.

\section{Ointment}

According to Rathore \& Nema (2009), ointment is a mixture of semisolid and solid hydrocarbon, such as paraffin, which is non-irritating to the eye and melts at body's physiological temperature. Commonly, there are two types of ointment, namely simple-based ointment which is made up of one continuous phase of ointment and compound-based ointment which consists of two-phase system like emulsion. When applied to the eye, the ointment will break into small drops that will remain in the conjunctival sac for a longer period of time (Baranowski, Karolewicz, Gajda, \& Pluta, 2014). This action leads to the major advantage of ointment, such that it serves as a drug depot in conjunctival sac which enhances and prolongs its absorption (Ghate \& Edelhauser, 2006).

According to Ali \& Lehmussaari (2006), the desirable attributes of ointment development should include several factors such that it needs to be non-irritating to the eye, uniform, easily manufactured, and it does not cause excessive blurred vision. Even though it can enhance and prolong drug absorption, ophthalmic ointment faces a major drawback that can reduce its efficacy. According to Sasaki et al. (1999), the application of ointment can lead to the blurring of vision and occasional irritation, resulting in low patient compliance. Due to this, it is usually being applied at night before sleep (Rathore \& Nema, 2009).

\section{Polymeric Gel}

Ocular gel is another dosage form of delivering drugs to the eye topically. Gels are made up of various materials such as mucoadhesive polymers which are important for the localised delivery of active ingredients. Mucoadhesive polymers have been used in ophthalmic gels to increase their efficacy (Shaikh, Raj Singh, Garland, Woolfson, \& Donnelly, 2011). This polymer provides an attachment for the drug carrier to a biological tissue resulting in an extended contact time and an improved ocular bioavailability (Ali \& Lehmussaari, 2006). There are two types of ophthalmic gels, namely preformed gel and in-situ forming gel. According to Ranch et al. (2017), ophthalmic preformed gel is less preferable as a dosage form because it is present as a gel substance at room temperature. This property has a limited use in ophthalmic drug delivery because of low accuracy and reproducibility administration of drugs, often producing blurry vision, crusty eyelids, and lachrymation. Due to this, in situ gels become a focus in gelling system as it provides both advantages of solution and gel.

In situ forming gel is a viscous liquid preparation that will change to a gel phase using either one of these three mechanisms which are $\mathrm{pH}$ triggered, temperature triggered, 
or ion activated. It is preferred over the preformed gel as it is more comfortable, easily administered as a drop, and causing less to no problem to the vision (Rathore, 2010). Kaur, Singh, \& Kanwar (2000) stated that good in situ forming gel criteria should include low viscosity, freeflowing property to be administered as a drop, and strong gel formation to withstand the sheer force of the conjunctiva. According to Gurtler \& Gurny (1995), it is difficult to administer accurate dose with preformed gel due to the variation of the amount of drug delivered during administration. However, with in situ gel-forming formulation, it is possible to administer accurate and reproducible quantities of dose. Moreover, relatively prolonged action duration of in situ forming gel reduces the administration frequency and thus increases patient compliance.

\section{Novel topical ocular drug delivery system}

Even though conventional topical ocular preparations are being widely used nowadays, some drawbacks are still present in terms of their usage, efficacy and safety. Due to these, various approaches have been made and studied. One of the approaches is by utilising nanotechnology in the ocular drug delivery system through nanoparticles and nanomicelles. There are also several other approaches to improve ocular delivery system such as liposomes and ocular inserts.

\section{Nanoparticles}

Sahoo, Dilnawaz, \& Krishnakumar (2008) defined nanoparticles as any particles with a diameter not bigger than one micrometer, comprising of various natural or synthetic polymers, lipids, phospholipids, or metals. There are two types of nanoparticles: nanocapsules and nanospheres. In nanocapsules, the drug is encapsulated inside the polymeric capsule while in nanospheres, the drug is uniformly dispersed throughout the polymeric matrix (Patel et al., 2013). One of the advantages of nanoparticles is that it can extend the drugs delivery to the tissues as the uptake and the distribution of nanoparticles depend on their size (Gaudana, Jwala, Boddu, $\&$ Mitra, 2009). This is proven in the study done by Sakurai, Ozeki, Kunou, \& Ogura (2001) on the significance of particle size in tissue distribution. The study concluded that the smaller particle size could be distributed further to the tissue area where no large particle is present.

Many other approaches have been developed using the nanoparticle technology. One of them is solid lipid nanoparticles. Solid lipid nanoparticles have several advantages such as they can improve corneal absorption, enhance corneal ocular bioavailability for both hydrophilic and lipophilic drug, allow autoclave sterilisation, and they do not display any biotoxicity since physiological lipids are used during the preparation process (Seyfoddin, Shaw, \& AlKassas, 2010). Other than that, solid lipid nanoparticles also show sustained drug release properties based on an in vivo study done by Cavalli, Gasco, Chetoni, Burgalassi, \& Saettone (2002). Solid lipid nanoparticles of tobramycin showed sustained drug release for up to six hours in comparison to short duration of tobramycin eye drops with an equal dose.

De Campos, Diebold, Carvalho, Sánchez, \& Alonso (2004) performed a study on chitosan fluorescent nanoparticles and found that the nanoparticles were stable upon incubation with lysozyme and did not affect the viscosity of mucin dispersion. The study found that the amount of chitosan fluorescent in cornea and conjunctiva were higher for the nanoparticles compared to the controlled chitosan fluorescent solution, and the amount was constant up to 24 hours. After 24 hours of incubation with chitosan nanoparticles, the cell survival was remarkable and the viability of the recovered cell was nearly 100 percent. Aside from that, there is also a study done by Motwani et al. (2008) on submicroscopic reservoir using nanoparticles. In this study, mucoadhesive chitosan-sodium alginate nanoparticles were used to deliver gatifloxacin to the eye. As a result, it was found that this system underwent a fast release for the first hour and continued on slow release for the rest of 24 hours study. The outcome reduces the frequency of dosing which then increases its patient compliance.

\section{Nanomicelles}

According to Patel et al. (2013), nanomicelles are the most frequently used carrier system to deliver therapeutic agents into clear aqueous solution. Nanomicelles are made up of amphiphilic molecules which are surfactants or polymers in nature that will self-assemble into micelles. There are three different types of micelles, namely regular micelles, reverse micelles, and unimolecular micelles (Trivedi \& Kompella, 2010). Regular micelles are amphiphilic copolymers which self-assemble in aqueous medium while reverse micelles are amphiphilic copolymers which self-assemble in non-aqueous medium. Unimolecular micelles on the other hand are made up of the block of copolymer which has several hydrophobic and hydrophilic regions in one molecule. This enables it to self-assemble into a micelle from one molecule. Between these three types, the reverse micelle is a good candidate to encapsulate and deliver hydrophilic drug as it forms micelles with the polar part facing towards the interior covering of the hydrophilic substances. In addition, Qiu, Zhang, Yan, Jin, \& Zhu (2007) mentioned that reverse micelles could also be used to encapsulate polymeric particles.

Nanomicelles pose several advantages as a drug delivery system. Nishiyama \& Kataoka (2006) pointed out that it requires a simple preparation and it has the ability to improve drug solubility, lower the toxicity, raise circulation time, and increase tissue penetration with well targeted delivery properties. In vivo studies on rabbits done by Civiale, Licciardi, Cavallaro, Giammona, \& Mazzone (2009) suggested that nanomicellar formulations are a better option for topical delivery of small molecules compared to 
suspension. In another study, Cholkar, Patel, Dutt Vadlapudi, \& K. Mitra (2012) concluded that nanomicellar formulations could efficiently transverse ocular tissues and deliver drug to the back of the eye tissues. However, the conventional micelles face a little drawback. It is not stable over a long period of time, having short period of sustained release, inadequate suitability for hydrophilic drugs, and system optimisation is needed for each drug (Torchilin, 2006). Thus, these need to be considered for improvement.

\section{Liposomes}

Liposomes have an aqueous core containing drug which is enclosed by one or more phospholipid bilayers. According to Patel et al. (2013), liposomes with the ability to encapsulate both hydrophobic and hydrophilic drugs can be classified into three types, namely small unilamellar vesicles $(10-100 \mathrm{~nm})$, large unilamellar vesicles $(100-300 \mathrm{~nm})$, and multilamellar vesicles (contain more than one bilayer). These liposomes are promising means in delivering ophthalmic drug due to the presence of natural phospholipids, cell-like membranes and excellent biocompatibility (Gan et al., 2013). Other than that, liposomes can attach to the hydrophobic corneal epithelium on which they constantly release the bound drug content, enhancing pharmacokinetics, and reducing the toxic side effect (Chetoni, Burgalassi, Monti, Najarro, \& Boldrini, 2007). Additionally, Budai et al. (2007) stated that sustained release of the drug could be produced by using multilamellar vesicles depending on the nature of the selected lipid composition.

A study using a rabbit model was performed by Shen \& $\mathrm{Tu}$ (2007), where they measured the concentration-time profile of ganciclovir in aqueous humour after been instilled with liposomes containing ganciclovir and ganciclovir solution. The results showed that the area under the curve for liposomes containing ganciclovir was 1.7 times bigger than the ganciclovir solution. The drug distribution of liposomal formulation was higher in sclera, cornea and vitreous humour. Another study was performed by Habib, Fouad, \& Fathalla (2008) comparing fluconazole solution and fluconazole loaded liposomes on the rabbit keratitis model. After 21 days, liposomal formulation was found successful at eliminating the infection and superior to the solution. These two studies clearly show that liposomal formulation is a better delivery system than solution. However, liposomes also suffer several drawbacks. This formulation tends to be unstable, degraded, and aggregated while its fuses cause leakage of entrapped drugs during storage and after administration (Zhang \& Wang, 2009). Thus, Mehanna, Elmaradny, \& Samaha (2010) suggested surface modification and polymerisation to be carried out to enhance the performance of liposomes.

\section{Ocular Inserts}

Ocular inserts are sterile preparation with a thin, multilayered and drug-impregnated solid or semisolid devices placed into conjunctival sac whose size and shape are especially designed for ophthalmic application (Kumari, Sharma, Garg, \& Garg, 2010). The main purpose of the ocular inserts is to improve the contact duration between the delivery system and the conjunctival tissue to ensure a prolonged release that suits topical or systemic treatment. According to Kumar, Bhowmik, Harish, Duraivel, \& Kumar (2013), there are two types of ocular inserts, namely soluble and insoluble ocular inserts. Soluble ocular inserts are generally defined as erodible, monolithic polymer that undergoes slow dissolution while releasing the drug and does not require removal from the eye. The insoluble type of ocular inserts is made up of insoluble polymer that can deliver drug by a variety of methods and at a predetermined rate, but it needs to be removed from the eye when empty.

Sultana, Jain, Aqil, \& Ali (2006) viewed the delivery of ocular inserts as more controlled, sustained and continuous. In doing so, it maintains an effective drug concentration in the target tissue and minimises the number of applications. However, based on the review, they found that the usage of this delivery system is less popular among users due to physiological factors such as patients' unwillingness to abandon the traditional liquid and semisolid medication, and occasional therapeutic failures such as unintentional expulsion from the eye and membrane rupture. Ocular inserts give several advantages such that they increase contact time, exhibit prolonged release, reduce systemic side effect, reduce dosing frequency, produce accurate dosing, increase shelf-life compared to aqueous solutions and elimination of preservative, thus leave less sensitivity reaction (Kumari et al., 2010). However, ocular inserts also come with their own disadvantages. The foreign-body sensation in the eye can lead to discomfort, causing low patient compliance, excessive lachrymation which accompanies with irritation, drug dilution, and concentration reduction (Friedrich, Saville, Cheng, \& Rootman, 1996). Kumari et al. (2010) also mentioned some other disadvantages of ocular inserts such as unwanted migration in the conjunctival sac, unintended loss, and difficulties to place or remove as well as interference with the vision.

\section{Conclusion}

There are various types of ocular drug delivery systems found in the literature and the market. Despite that, drug delivery remains a conundrum and major challenge for ocular and formulation scientists due to the complexity of the eye structure. Until now, topical eye drops remain the most preferred approach for eye treatment especially for the anterior application due to the ease of administration. However, eye drops formulation faced several major drawbacks that can reduce its efficacy such as loss of active agents by tear drainage, low corneal permeability, and reduced patient compliance following frequent administration. Due to these, several conventional ocular drug delivery systems have been developed as options to improve treatment efficacy. The examples are ocular 
emulsion, suspension, ointment and polymeric gels. Aside from these conventional delivery systems, scientists are developing more ocular delivery systems such as nanomicelles, nanoparticles, liposomes and ocular inserts. These novel systems are developed to further increase the efficacy and safety in the application of ocular drug delivery. Despite that, there still possessed several drawbacks. It is hoped that the future novel systems would be able to overcome all the drawbacks while retains its efficacy, safety and improve patient compliance.

\section{Acknowledgements}

This work was supported by the International Islamic University Malaysia Research Initiative Grant Scheme (RIGS 16-288-0452).

\section{Conflict of Interest}

None.

\section{References}

Aldrich, D. S., Bach, C. M., Brown, W., Chambers, W., Fleitman, J., Hunt, D., \& Tin, G. W. (2013). Ophthalmic preparations. USP Council of Experts, 39(5).

Ali, Y., \& Lehmussaari, K. (2006). Industrial perspective in ocular drug delivery. Advanced Drug Delivery Reviews, 58(11), 1258-1268.

Baranowski, P., Karolewicz, B., Gajda, M., \& Pluta, J. (2014). Ophthalmic drug dosage forms: Characterisation and research methods. The Scientific World Journal, 2014, 14p.

Budai, L., Hajdú, M., Budai, M., Gróf, P., Béni, S., Noszál, B., Klebovich, I., \& Antal, I. (2007). Gels and liposomes in optimized ocular drug delivery: Studies on ciprofloxacin formulations. International Journal of Pharmaceutics, 343(1-2), 34-40.

Cavalli, R., Gasco, M. R., Chetoni, P., Burgalassi, S., \& Saettone, M. F. (2002). Solid lipid nanoparticles (SLN) as ocular delivery system for tobramycin. International Journal of Pharmaceutics, 238(1-2), 241-245.

Chetoni, P., Burgalassi, S., Monti, D., Najarro, M., \& Boldrini, E. (2007). Liposome-encapsulated mitomycin $\mathrm{C}$ for the reduction of corneal healing rate and ocular toxicity. Journal of Drug Delivery Science and Technology, 17(1), 43-48.

Cholkar, K., Patel, A., Dutt Vadlapudi, A., \& K. Mitra, A. (2012). Novel Nanomicellar Formulation Approaches for Anterior and Posterior Segment Ocular Drug Delivery. Recent Patents on Nanomedicinee, 2(2), 8295.
Civiale, C., Licciardi, M., Cavallaro, G., Giammona, G., \& Mazzone, M. G. (2009). Polyhydroxyethylaspartamide-based micelles for ocular drug delivery. International Journal of Pharmaceutics, 378(1-2), 177-186.

De Campos, A. M., Diebold, Y., Carvalho, E. L. S., Sánchez, A., \& Alonso, M. J. (2004). Chitosan nanoparticles as new ocular drug delivery systems: In vitro stability, in vivo fate, and cellular toxicity. Pharmaceutical Research, 21(5), 803-810.

Friedrich, S. W., Saville, B. A., Cheng, Y. L., \& Rootman, D. S. (1996). Pharmacokinetic differences between ocular inserts and eyedrops. Journal of Ocular Pharmacology and Therapeutics, 21(1), 5-18.

Gan, L., Wang, J., Jiang, M., Bartlett, H., Ouyang, D., Eperjesi, F., Liu, J., \& Gan, Y. (2013). Recent advances in topical ophthalmic drug delivery with lipid-based nanocarriers. Drug Discovery Today, 18(5-6), 290-297.

Gaudana, R., Jwala, J., Boddu, S. H. S., \& Mitra, A. K. (2009). Recent perspectives in ocular drug delivery. Pharmaceutical Research, 26(5), 1197-1216.

Ghate, D., \& Edelhauser, H. F. (2006). Ocular drug delivery. Expert Opinion on Drug Delivery, 3(2), 275-287.

Gurtler, F., \& Gurny, R. (1995). Patent literature review of ophthalmic inserts. Drug Development and Industrial Pharmacy, 21(1), 1-18.

Habib, F. S., Fouad, E. A., \& Fathalla, D. (2008). Liposomes as an ocular delivery system of fluconazole: In-vitro studies. Bulletin of Pharmaceutical Sciences, 88(8), 901-904.

Kaur, I. P., Singh, M., \& Kanwar, M. (2000). Formulation and evaluation of ophthalmic preparations of acetazolamide. International Journal of Pharmaceutics, 199(2), 119-127.

Kinoshita, S., Awamura, S., Oshiden, K., Nakamichi, N., Suzuki, H., \& Yokoi, N. (2012). Rebamipide (OPC12759) in the treatment of dry eye: A randomized, double-masked, multicenter, placebo-controlled phase II study. Ophthalmology, 119(12), 2471-2478.

Kumar, K. P., Bhowmik, D., Harish, G., Duraivel, S., \& Kumar, B. (2013). Ocular inserts: a novel controlled drug delivery system. The Pharma Innovation, 1(12), $1-16$.

Kumari, A., Sharma, P. K., Garg, V. K., \& Garg, G. (2010). Ocular inserts-Advancement in therapy of eye diseases. Journal of Advanced Pharmaceutical Technology and Research, 1(3), 291-296. 
Liang, H., Brignole-Baudouin, F., Rabinovich-Guilatt, L., Mao, Z., Riancho, L., Faure, M. O., Warnet, J. M., Lambert, G. \& Baudouin, C. (2008). Reduction of quaternary ammonium-induced ocular surface toxicity by emulsions: An in vivo study in rabbits. Molecular Vision, 14, 204-216.

Mehanna, M. M., Elmaradny, H. A., \& Samaha, M. W. (2010). Mucoadhesive liposomes as ocular delivery system: Physical, microbiological, and in vivo assessment. Drug Development and Industrial Pharmacy, 36(1), 108-118.

Monkhouse, S. (2007). Clinical Anatomy: A Core Text with Self-assessment. London, United Kingdom: Churchill Livingstone.

Motwani, S. K., Chopra, S., Talegaonkar, S., Kohli, K., Ahmad, F. J., \& Khar, R. K. (2008). Chitosan-sodium alginate nanoparticles as submicroscopic reservoirs for ocular delivery: Formulation, optimisation and in vitro characterisation. European Journal of Pharmaceutics and Biopharmaceutics, 68(3), 513-525.

Nishiyama, N., \& Kataoka, K. (2006). Current state, achievements, and future prospects of polymeric micelles as nanocarriers for drug and gene delivery. Pharmacology and Therapeutics, 112(3), 630-648.

Pahuja, P., Arora, S., \& Pawar, P. (2012). Ocular drug delivery system: A reference to natural polymers. Expert Opinion on Drug Delivery, 9(7), 837-861.

Patel, A., Cholkar, K., Agrahari, V., \& Mitra, A. K. (2013). Ocular drug delivery systems: An overview. World Journal of Pharmacology, 2(2), 47-64.

Qiu, L., Zhang, J., Yan, M., Jin, Y., \& Zhu, K. (2007). Reverse self-assemblies based on amphiphilic polyphosphazenes for encapsulation of water-soluble molecules. Nanotechnology, 18(47), 9p.

Ranch, K., Patel, H., Chavda, L., Koli, A., Maulvi, F., \& Parikh, R. K. (2017). Development of in situ ophthalmic gel of dexamethasone sodium phosphate and chloramphenicol: A viable alternative to conventional eye drops. Journal of Applied Pharmaceutical Science, 7(3), 101-108.

Rathore, K. S. (2010). In-situ gelling ophthalmic drug delivery system: An overview. International Journal of Pharmacy and Pharmaceutical Sciences, 9(3), 237248.

Rathore, K. S., \& Nema, R. K. (2009). An Insight into Ophthalmic Drug Delivery System. International Journal of Pharmaceutical Sciences and Drug Research, 1(1), 1-5.
Remington, J. P. (2011). Remington: The Science and Practice of Pharmacy. Maryland, USA: Lippincot Williams \& Wilkins.

Sahoo, S. K., Dilnawaz, F., \& Krishnakumar, S. (2008). Nanotechnology in ocular drug delivery. Drug Discovery Today, 13(3-4), 144-151.

Sakurai, E., Ozeki, H., Kunou, N., \& Ogura, Y. (2001). Effect of particle size of polymeric nanospheres on intravitreal kinetics. Ophthalmic Research, 33(1), 3136.

Sasaki, H., Yamamura, K., Mukai, T., Nishida, K., Nakamura, J., Nakashima, M., \& Ichikawa, M. (1999). Enhancement of ocular drug penetration. Critical Reviews in Therapeutic Drug Carrier Systems, 16(1), 85-146.

Scoper, S. V., Kabat, A. G., Owen, G. R., Stroman, D. W., Kabra, B. P., Faulkner, R., Kulshreshtha, A. K., Rusk, C., Bell, B., Jamison, T., Bernal-Perez, L. F., Brooks, A. C. \& Nguyen, V. A. (2008). Ocular distribution, bactericidal activity and settling characteristics of TobraDex ${ }^{\circledR}$ ST ophthalmic suspension compared with TobraDex ${ }^{\circledR}$ ophthalmic suspension. Advances in Therapy, 25(2), 77-88.

Seyfoddin, A., Shaw, J., \& Al-Kassas, R. (2010). Solid lipid nanoparticles for ocular drug delivery. Drug Delivery, 17(7), 467-489.

Shaikh, R., Raj Singh, T. R., Garland, M. J., Woolfson, A. D., \& Donnelly, R. F. (2011). Mucoadhesive drug delivery systems. Journal of Pharmacy \& Bioallied Sciences, 3(1), 89-100.

Shen, Y., \& Tu, J. (2007). Preparation and ocular pharmacokinetics of ganciclovir liposomes. AAPS Journal, 9(3), E371-E377.

Souza, J. G., Dias, K., Pereira, T. A., Bernardi, D. S., \& Lopez, R. F. V. (2014). Topical delivery of ocular therapeutics: Carrier systems and physical methods. Journal of Pharmacy and Pharmacology, 66(4), 507530.

Sultana, Y., Jain, R., Aqil, M., \& Ali, A. (2006). Review of ocular drug delivery. Current Drug Delivery, 3(2), 207217.

Torchilin, V. P. (2006). Micellar Nanocarriers: Pharmaceutical Perspectives. Pharmaceutical Research, 24(1), 1. 
Tortora, G. J., \& Derrickson, B. H. (2015). Principles of Anatomy and Physiology. Queensland, Australia: John Wiley \& Sons.

Trivedi, R., \& Kompella, U. B. (2010). Nanomicellar formulations for sustained drug delivery: Strategies and underlying principles. Nanomedicine, 5(3), 485-505.

Vandamme, T. F. (2002). Microemulsions as ocular drug delivery systems: Recent developments and future challenges. Progress in Retinal and Eye Research, 21(1), 15-34.

Yamaguchi, M., Ueda, K., Isowaki, A., Ohtori, A., Takeuchi, H., Ohguro, N., \& Tojo, K. (2009). Mucoadhesive properties of chitosan-coated ophthalmic lipid emulsion containing indomethacin in tear fluid. Biological and Pharmaceutical Bulletin, 32(7), 12661271.

Zhang, J., \& Wang, S. (2009). Topical use of Coenzyme Q10-loaded liposomes coated with trimethyl chitosan: Tolerance, precorneal retention and anti-cataract effect. International Journal of Pharmaceutics, 372(1-2), 6675. 